\title{
Choline- and acetylcholine-induced changes in the morphology of Fusarium graminearum : evidence for the involvement of the choline transport system and acetylcholinesterase
}

\author{
Geoffrey D. Robson, Marilyn G. Wiebe, Bryan Cunliffe \\ and Anthony P. J. Trinci
}

Author for correspondence: Geoffrey D. Robson. Tel: +44161275 5048. Fax: +441612755656.

Microbiology Research Group, 1.800 Stopford Building, School of Biological Sciences, University of Manchester Manchester M13 9PT, UK

\begin{abstract}
The response of Fusarium graminearum to choline, acetylcholine and a number of related analogues was investigated and their ability to induce a morphological response quantified. A number of mutants resistant to the alkylating agent nitrogen mustard (nim strains) were generated and found to have lost the ability to transport choline. These mutants were found to be insensitive to choline and acetylcholine but not to betaine, ethanolamine and other analogues. In addition, the non-competitive inhibitor hemicholinium-3 was also found to reduce the morphological effect of choline, proving that transport of choline into the hypha is essential for the morphological response. Acetylcholinesterase inhibitors blocked the morphological response to acetylcholine but had no effect on the response to choline, suggesting the presence of a membrane- or wall-bound acetylcholinesterase that hydrolyses acetylcholine to choline which subsequently induces the morphological response. Studies on the in vivo chitin synthase activity revealed that addition of choline caused a transient $75 \%$ increase in chitin synthase activity within $30 \mathrm{~s}$, the rate rapidly returning to that observed before the addition of choline. No such effect was observed with the nim mutants.
\end{abstract}

Keywords: Fusarium graminearum, choline, hyphal growth, nitrogen mustard, chitin synthase

\section{INTRODUCTION}

Choline and the related analogue betaine, were isolated from wheat anthers and identified as virulence enhancers for Fusarium graminearum (Strange \& Smith, 1971; Strange et al., 1974). Later they were found to cause an increase in the spread of the mycelium due to stimulation of the hyphal extension rate, although the overall growth rate remained unchanged (Wiebe et al., 1989). A number of other related compounds, including phosphorylcholine, ethanolamine and acetylcholine, have also been shown to stimulate hyphal extension in F. graminearum to differing degrees (Strange \& Smith, 1978; Wiebe et al., 1989).

Recently, we have characterized a constitutive choline (Robson et al., 1992) and a constitutive betaine (Robson et al., 1994) transport system in F. graminearum, which may be involved in mediating the morphological effects of

Abbreviations: 1,5(4-ADAP), 1,5-bis(4-allyldimethyl-ammoniumphenyl)pentan-3-one dibromide; GICNAC, $\mathrm{N}$-acetylglucosamine. these two compounds. Previously however, it has not been possible to distinguish whether the morphological effect of choline on F. graminearum acted externally or required transport into the hypha. In Saccharomyces cerevisiae, resistance of mutants to the alkylating agent nitrogen mustard has been found to be due to the loss of a single recessive gene encoding choline permease ( $\mathrm{Li}$ et al., 1991), resulting in the inability of these mutants to transport choline.

In this paper, we quantify the stimulatory effects of choline and related analogues on the colony radial growth rate of $F$. graminearum A3/5. In addition, we have isolated a number of nitrogen-mustard-resistant mutants which lack the ability to transport choline, and have investigated the morphological effects of choline and related analogues on these mutants.

\section{METHODS}

Organism, media and chemicals. F. graminearum strain A3/5 was obtained from $\mathrm{Mr} \mathrm{T}$. W. Naylor, (Marlow Foods, 
Billingham, UK) and maintained in $20 \%(\mathrm{v} / \mathrm{v})$ glycerol at $-80^{\circ} \mathrm{C}$.

All cultures were grown on modified Vogel's medium (Vogel, 1956) in which $10 \mathrm{~g} \mathrm{D}$-glucose $\mathrm{l}^{-1}$ replaced sucrose as the carbon source. For liquid cultures, $F$. graminearum A3/5 was grown in $50 \mathrm{ml}$ Vogel's medium in $250 \mathrm{ml}$ flasks on a rotary shaker (200 r.p.m.) and inoculated with $1 \mathrm{ml}$ of a $1 \times 10^{6}$ spore $\mathrm{ml}^{-1}$ suspension derived from a 7-10 d-old culture. For agar medium, $1.5 \%(\mathrm{w} / \mathrm{v}$ ) Tayo agar (Lucas Meyer) was added. All cultures were incubated at $25^{\circ} \mathrm{C}$.

All additions to the medium were prepared as filter-sterilized $(0.22 \mu \mathrm{m}$ pore size, Whatman) stock solutions in distilled water (DMSO for hemicholinium-3) and stored in aliquots at $-20^{\circ} \mathrm{C}$ until required. Stock solutions were diluted $\times 100$ to give the final concentration required in the medium and added either to molten agar at $55^{\circ} \mathrm{C}$, or to liquid medium at room temperature. $\left[{ }^{3} \mathrm{H}\right] N$-Acetylglucosamine (GlcNAc) was obtained from Amersham (sp. act. $7 \cdot 8 \mathrm{Ci} \mathrm{mmol}^{-1}$ ). All chemicals were obtained from Sigma unless otherwise stated.

Colony radial growth rate determinations. Colony diameters were measured twice daily up to $96 \mathrm{~h}$ after inoculation in two $90^{\circ}$ planes on four replicate colonies on plates which had been inoculated centrally with a $2.5 \mu \mathrm{l}$ drop of a $1 \times 10^{6}$ spore $\mathrm{ml}^{-1}$ suspension. Colony radial growth rates $\left(K_{\mathrm{r}}\right)$ (Trinci, 1971) were calculated from the slope of the line from a plot of colony radius vs time since inoculation, by least squares regression analysis. Isolation of nitrogen-mustard-resistant (nim) mutants. An aliquot $(0 \cdot 1 \mathrm{ml})$ of a $1 \times 10^{8}$ spore $\mathrm{ml}^{-1}$ suspension was placed on Vogel's agar medium containing $100 \mu \mathrm{g}$ nitrogen mustard $\mathrm{ml}^{-1}$. The nim mutants were isolated as colonies that grew vigorously on the plates and appeared between 4 and $8 \mathrm{~d}$ after inoculation. The nim mutants were subcultured on nitrogen mustard agar to check their validity.

Choline transport assay. Uptake of $\left[{ }^{14} \mathrm{C}\right]$ choline by $F$. graminearum A3/5 and nim mutants was performed as described previously (Robson et al., 1992).

Incorporation of $\left[^{3} \mathrm{H}\right] \mathrm{GlcNAc}$ by mycelia. Incorporation of $\left[\mathrm{H}^{3}\right] \mathrm{GlcN} A \mathrm{c}$ into the fungal biomass was carried out as described previously (Binks et al., 1990). $\left[{ }^{3} \mathrm{H}\right] \mathrm{GlcN} A c$ $\left(0.08 \mu \mathrm{Ci} \mathrm{ml} \mathrm{m}^{-1}\right)$ was added to an exponentially growing culture of $F$. graminearum. Duplicate $0.5 \mathrm{ml}$ samples were removed at 1,2 or $3 \mathrm{~min}$ intervals and added to $0.5 \mathrm{ml} 10 \%$ (w/v) trichloroacetic acid. Samples were then filtered through Whatman no. 1 filter papers and washed with three $2 \mathrm{ml}$ volumes of distilled water. The biomass and filters were then added to scintillation vials containing $4 \mathrm{ml}$ scintillation fluid (Optiphase Hisafe) and radioactivity counted using a Packard Tricarb 1500 Scintillation counter. Choline $(20 \mu \mathrm{M}$ final concentration) was added to the culture from a $\times 100$ stock, $15 \mathrm{~min}$ after the addition of $\left[{ }^{3} \mathrm{H}\right] \mathrm{GlcN} A \mathrm{c}$.

\section{RESULTS}

\section{Effect of choline and related analogues on the morphology of $F$. graminearum A3/5}

A typical dose-response curve for the effects of choline on the $K_{\mathrm{r}}$ of $F$. graminearum is shown in Fig. 1a. The maximum stimulation in $K_{\mathrm{r}}$ was calculated as the percentage increase in $K_{\mathrm{r}}$ compared with the control at the point where a further increase in the choline concentration had no additional effect on $K_{\mathrm{r}}$. Probit transformation of the curve produced a linear relationship and therefore the concentration which gave a $5 \%\left(\mathrm{ED}_{5}\right)$ or $50 \%\left(\mathrm{ED}_{50}\right)$ increase in the $K_{\mathrm{r}}$ was calculated (Fig. 1b). Using this

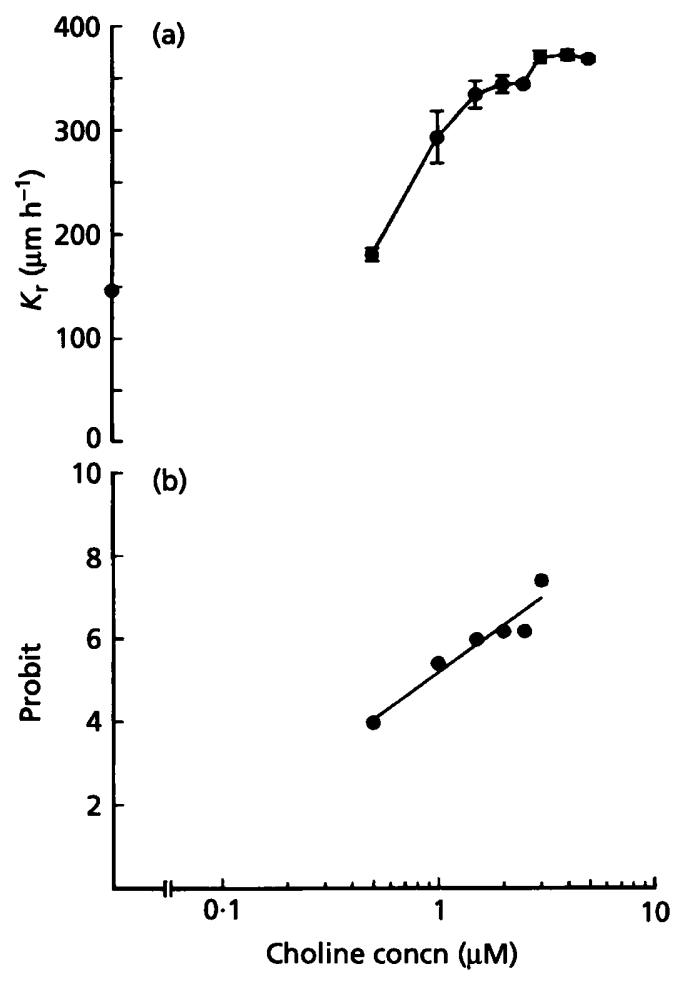

Fig. 1. (a) Effect of various choline concentrations on the colony radial growth rate of $F$. graminearum A3/5. (b) The same data transformed as a probit plot; $\mathrm{LD}_{50}, 0.9 \mu \mathrm{M}$, Max., $154 \%$; $\mathrm{LD}_{5}, 0.3 \mu \mathrm{M}$.

Table 1. Effect of choline and related analogues on the colony radial growth rate of $F$. graminearum

1-Amino-propan-2-ol, triethanolamine, acetylthiocholine and carbamylcholine had no effect up to a concentration of $1 \mathrm{mM}$.

\begin{tabular}{|lccc|}
\hline Compound & $\mathbf{E D}_{\mathbf{5}}(\boldsymbol{\mu m}) \mathbf{E D}_{\mathbf{5 0}}(\boldsymbol{\mu M})$ & $\begin{array}{c}\text { Percentage } \\
\text { increase } \\
\text { in } \mathbf{K}_{\mathbf{r}}^{*}\end{array}$ \\
\hline Choline & & & 154 \\
Ethanolamine & $0 \cdot 3$ & $0 \cdot 9$ & 162 \\
Monomethylethanolamine & $1 \cdot 4$ & $5 \cdot 0$ & 155 \\
Dimethylethanolamine & $0 \cdot 9$ & 85 & 146 \\
Phosphorylethanolamine & 32 & 148 & 162 \\
Betaine & $0 \cdot 3$ & $5 \cdot 4$ & 106 \\
Acetylcholine & $4 \cdot 4$ & 127 & 146 \\
Propionylcholine & 73 & 177 & 110 \\
Butyrylcholine & 175 & 360 & 109 \\
Phosphorylcholine & 4 & 30 & 154 \\
Chlorocholine & 25 & 126 & 90 \\
3-Amino-1-propanol & 8 & 29 & 54 \\
\hline
\end{tabular}

$*$ Calculated as $100 \times \frac{K_{\mathrm{r}}(+ \text { additive })-K_{\mathrm{r}}(- \text { additive })}{K_{\mathrm{r}}(- \text { additive })}$.

approach, it was possible to quantify and compare the effects of the other compounds with that of choline (Table 1). With the exception of betaine, propionylcholine, 


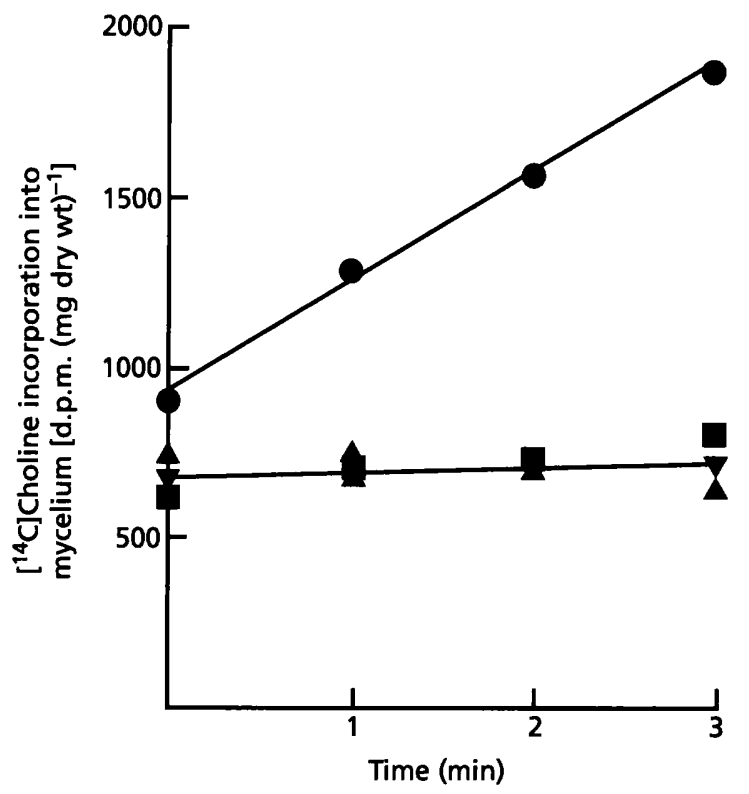

Fig. 2. Uptake of $\left[{ }^{14} \mathrm{C}\right]$ choline by $F$. graminearum $A 3 / 5$ (O), $\operatorname{nim} 1(\boldsymbol{D}), \operatorname{nim} 2(\boldsymbol{\Lambda})$ and $\operatorname{nim} 3(\boldsymbol{\nabla})$ mutants.

butyrylcholine, chlorocholine and 3-amino-1-propanol, all of the compounds which were effective stimulated $K_{\mathrm{r}}$ to approximately the same degree. However, there were substantial differences in the concentrations at which each of the compounds first began to stimulate $K_{\mathrm{r}}\left(\mathrm{ED}_{5}\right)$ and in the concentrations which gave half the maximal effect $\left(\mathrm{ED}_{50}\right)$. Choline and betaine caused morphological effects at the lowest concentrations of all of the compounds examined $(0.3 \mu \mathrm{M})$ whilst butyrylcholine required the highest concentration $(175 \mu \mathrm{M})$ to produce an effect. Similarly, the $\mathrm{ED}_{50}$ for choline $(0.9 \mu \mathrm{M})$ was lower than for the other compounds tested and the $\mathrm{ED}_{50}$ for butyrylcholine $(360 \mu \mathrm{M})$ was the highest. In general, those compounds which caused a morphological effect at higher concentrations, also caused maximum effect at the higher concentrations. There was little correlation between the maximum percentage increase in $K_{\mathrm{r}}$ and the $\mathrm{ED}_{5}$ or $\mathrm{ED}_{50}$ values.

\section{Response of nim mutants to choline}

Ten nim strains of $F$. graminearum A3/5 were isolated and found to grow weakly on agar medium when choline was supplied as the sole nitrogen source, compared with the vigorous growth observed with strain $\mathrm{A} 3 / 5$ under these conditions (results not shown). Choline uptake studies confirmed that nim mutants were unable to transport choline compared with the parental strain (Fig. 2). When Vogel's agar medium was supplemented with choline or related analogues, nim mutants showed a loss of or severely reduced morphological response to choline and acetyl choline and a reduced response to dimethylethanolamine. There was no significant difference in the response to ethanolamine, monomethylethanolamine or

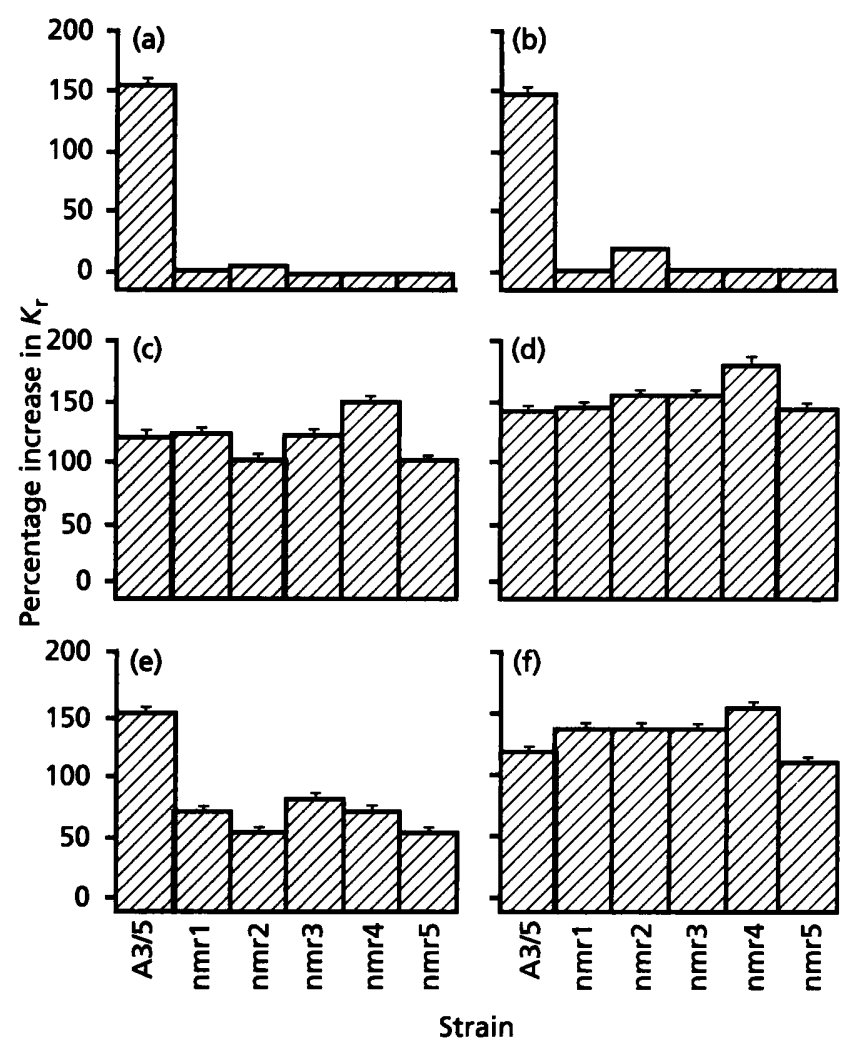

Fig. 3. Percentage stimulation of the colony radial growth rate of wild type and nim mutants of $F$. graminearum by $100 \mu \mathrm{M}$ choline (a), acetylcholine (b), ethanolamine (c), monomethylethanolamine (d), dimethylethanolamine (e) and betaine (f). Percentage stimulation of the colony radial growth rate was calculated as

$100 \times \frac{K_{\mathrm{r}}(+ \text { additive })-K_{\mathrm{r}}(- \text { additive })}{K_{\mathrm{r}}(- \text { additive })}$.

Results represent the mean of eight replicates with standard error bars.

betaine between the parental strain and the nim mutants (Fig. 3).

\section{Effect of hemicholinium-3 and acetylcholinesterase inhibitors on the morphological response of F. graminearum A3/5}

Hemicholinium-3 has been shown to act as a mixed noncompetitive inhibitor of choline uptake (Kinney \& Moore, 1988) and to block choline uptake by $F$. graminearum (Robson et al., 1992). Hemicholinium-3 reduced the morphological response of $F$. graminearum to $5 \mu \mathrm{M}$ choline by $30 \%$ at $1 \mu \mathrm{M}, 87 \%$ at $10 \mu \mathrm{M}$ and $95 \%$ at $100 \mu \mathrm{M}$ hemicholinium-3 concentrations (Table 2). At a concentration of $20 \mu \mathrm{M}$ choline, hemicholinium-3 was less effective in reducing the morphological response to choline. Hemicholinium-3 had no significant effect on the $K_{\mathrm{r}}$ in the absence of choline. In the presence of the acetylcholinesterase inhibitors neostigmine, 1,5-bis(4allyldimethyl-ammoniumphenyl)pentan-3-one dibromide 
Table 2. Effect of hemicholinium-3 on the colony radial growth rate of $F$. graminearum $A 3 / 5$ in the presence and absence of choline

Values are the mean of eight replicates \pm SEM.

\begin{tabular}{|cccc|}
\hline \multirow{2}{*}{$\begin{array}{c}\text { Hemicholinium-3 } \\
\text { concentration }(\boldsymbol{\mu M})\end{array}$} & \multicolumn{3}{c|}{$\boldsymbol{K}_{\mathrm{r}}\left(\boldsymbol{\mu} \mathrm{m} \mathrm{h}^{\mathbf{- 1}}\right)$} \\
\cline { 2 - 4 } & No addition & $\mathbf{5} \boldsymbol{\mu} \mathbf{M}$ choline & $\mathbf{2 0} \boldsymbol{\mu M}$ choline \\
\hline 0 & $149 \pm 3$ & $367 \pm 3$ & $359 \pm 6$ \\
1 & $145 \pm 4$ & $305 \pm 5$ & $360 \pm 3$ \\
10 & $148 \pm 2$ & $185 \pm 2$ & $310 \pm 3$ \\
100 & $147 \pm 2$ & $154 \pm 2$ & $195 \pm 4$ \\
\hline
\end{tabular}

Table 3. Effect of various acetylcholinesterase inhibitors on the morphological response of $F$. graminearum to choline and acetylcholine

Values are the mean of eight replicates \pm sEM.

\begin{tabular}{|lcccc|}
\hline Inhibitor & $\begin{array}{c}\text { Concentration } \\
(\boldsymbol{\mu} \mathbf{M})\end{array}$ & No additive & $\begin{array}{c}\mathbf{2 0} \boldsymbol{\mu} \mathbf{M} \\
\text { choline }\end{array}$ & $\begin{array}{c}\mathbf{1 0 0} \boldsymbol{\mu} \mathbf{M} \\
\text { acetylcholine }\end{array}$ \\
\cline { 3 - 4 } & & & $390 \pm 3$ & $301 \pm 8$ \\
& - & $149 \pm 2$ & $396 \pm 3$ & $270 \pm 11$ \\
None & 10 & $149 \pm 2$ & $402 \pm 5$ & $258 \pm 6$ \\
Neostigmine & 100 & $148 \pm 6$ & $399 \pm 4$ & $194 \pm 6$ \\
& 1000 & $150 \pm 2$ & $385 \pm 8$ & $180 \pm 2$ \\
$1,5(4-\mathrm{ADAP})$ & 1 & $147 \pm 4$ & $391 \pm 8$ & $155 \pm 2$ \\
& 10 & $143 \pm 2$ & $219 \pm 9$ & $147 \pm 2$ \\
& 100 & $153 \pm 2$ & - & $303 \pm 13$ \\
Eserine & 10 & $157 \pm 2$ & - & $306 \pm 13$ \\
& 100 & $153 \pm 4$ & $165 \pm 5$ \\
\hline
\end{tabular}

[1,5(4-ADAP)] and eserine, the morphological response of $F$. graminearum $A 3 / 5$ to acetylcholine was progressively reduced with increasing concentrations of inhibitors (Table 3). At a concentration of $1 \mathrm{mM}$, neostigmine and eserine reduced the morphological response of $F$. graminearum $\mathrm{A} 3 / 5$ to acetylcholine by $72 \%$ and $92 \%$, respectively. 1,5(4-ADAP) completely blocked the morphological response to acetylcholine at a concentration of $10 \mu \mathrm{M}$. None of the inhibitors had any effect on the $K_{\mathrm{r}}$ of F. graminearum A3/5 with the exception of $100 \mu \mathrm{M} 1,5$ (4-ADAP).

\section{Effect of choline on the in vivo incorporation of $\left[H^{3}\right]$ GICNAc into the cell wall}

Incorporation of $\left[{ }^{3} \mathrm{H}\right] \mathrm{GlcNAc}$ into the cell wall of growing cultures of $F$. graminearum was approximately log linear up to $45 \mathrm{~min}$ (results not shown). Addition of choline, $15 \mathrm{~min}$ after the addition of radiolabel led to a rapid increase in the incorporation of radiolabel within $30 \mathrm{~s}$, representing a $75 \%$ increase in incorporation. Subsequently, incorporation continued at the same rate as that observed prior to the addition of choline (Fig. 4). No such effect was found when choline was added to growing cultures of the nim 1 mutant (results not shown).

\section{DISCUSSION}

It has been demonstrated previously that intermediates in the pathway from ethanolamine to betaine were capable of stimulating hyphal extension and thus $K_{\mathrm{r}}$ without affecting the overall growth rate of $F$. graminearum, leading to the development of a more rapidly expanding, sparsely branched mycelium (Wiebe et al., 1989). The stimulatory effects of these intermediates on $K_{\mathrm{r}}$ are quantified in Table 1 , demonstrating that the active compounds are all components of the pathway from ethanolamine to betaine (Fig. 5). Modifications to these compounds, e.g. the addition of a chloro- or phospho- group to choline or the replacement of a propyl or butyryl group for the acetyl group in acetylcholine, all lead to an increase in the concentration required to elicit the morphological response, probably due to a reduction in the rate of uptake. The relative concentration required to initiate a significant stimulation of extension (we arbitrarily chose the con- 


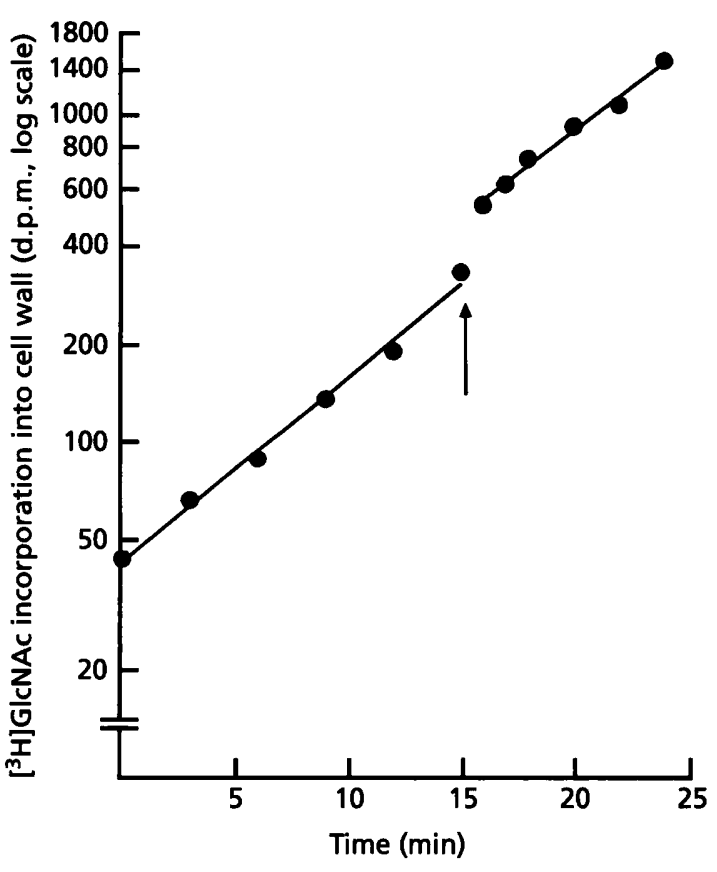

Fig. 4. Effect of the addtion of choline (arrow, $20 \mu \mathrm{M}$ final concentration) on the incorporation of $\left[{ }^{3} \mathrm{H}\right] \mathrm{GICNAC}$ into the cell wall of a growing culture of $F$. graminearum.

centration causing a $5 \%$ stimulation in $K_{\mathrm{r}}$ for comparisons) showed a broad range, with the lowest concentrations required for choline and betaine $(0.3 \mu \mathrm{M})$ and the highest for butyrylcholine and propionylcholine (175 and $73 \mu \mathrm{M}$, respectively). In general, the higher the concentration required to first elicit a morphological response, the higher the concentration required before the maximum response was reached.

F. graminearum A3/5 mutants, resistant to nitrogen mustard (nim), produced sparse growth when grown on medium containing choline as the sole nitrogen source. The growth observed was typical of nitrogen-starved mycelia, indicating the loss of choline uptake ability. Transport studies with radiolabelled choline confirmed a loss of choline uptake in these mutants (Fig. 2). nim mutants were found to have lost the morphological response to choline (Fig. 3), suggesting that the morphological effects are not due to external receptor-binding but that choline or a product thereof, acts inside the hypha. This view is further supported by the reduction of the response to choline by addition of hemicholinium- 3 , which acts as a non-competitive inhibitor of choline uptake (Table 2).

In addition to loss of sensitivity to choline, nim mutants also lost sensitivity to acetylcholine. Acetylcholine does not appear to be transported by the choline permease (Robson et al., 1992), suggesting that acetylcholine may first be hydrolysed to choline by an acetylcholinesterase located on the outside of the hypha, with the choline released eliciting the observed morphological effect. This conclusion is further supported by the loss of sensitivity

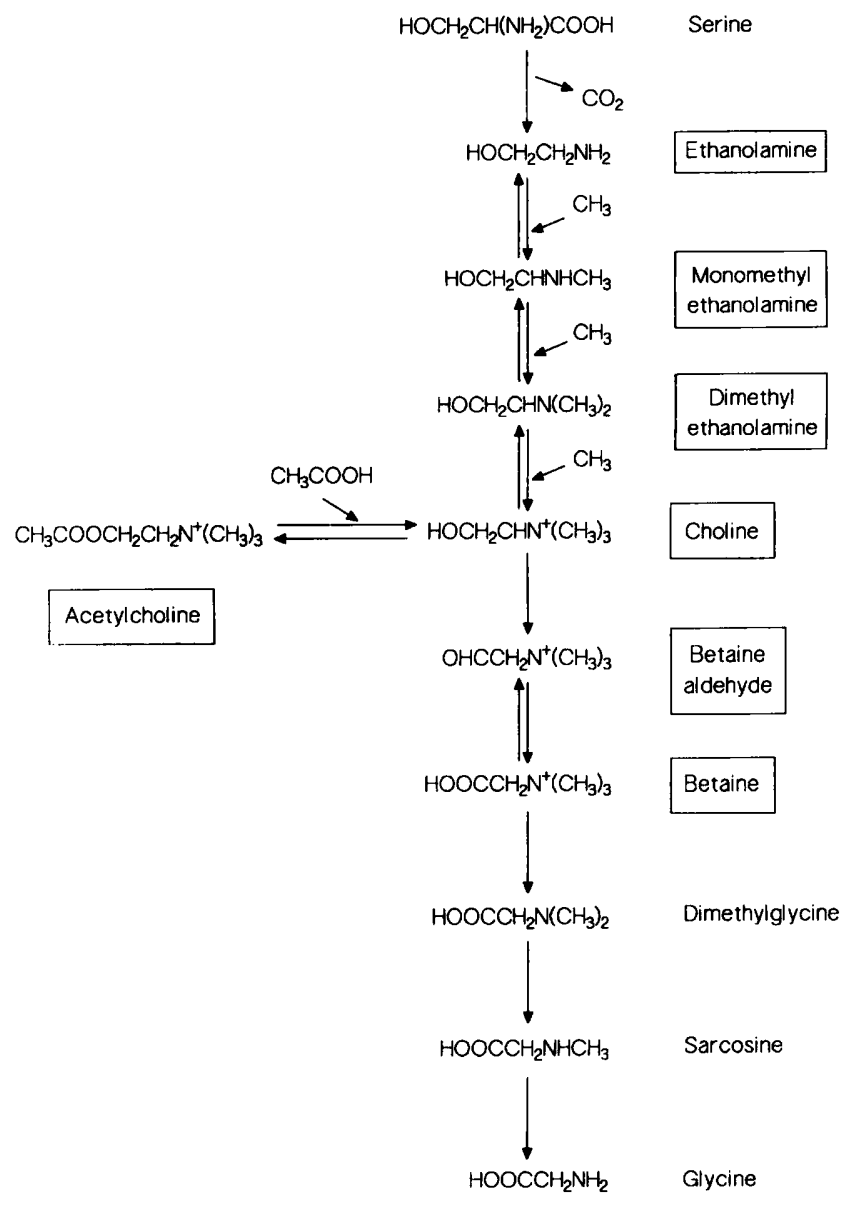

Fig. 5. Biosynthetic pathway of choline and betaine synthesis. Boxed compounds are capable of eliciting a morphological effect in F. graminearum.

to acetylcholine, but not to choline, in the presence of various acetylcholinesterase inhibitors (Table 3).

The morphological response of nim mutants to betaine was similar to that observed in the parental strain, confirming our previous report that betaine uptake occurs by a transport mechanism independent of choline (Robson et al., 1994). Similarly, there was no reduction in the degree of stimulation of nim mutants in the presence of ethanolamine, which we previously found did not compete for either the choline or betaine permease. A third permease capable of transporting ethanolamine may therefore exist. If it is also capable of transporting monomethylethanolamine and dimethylethanolamine, this third permease would explain why the response of $\mathrm{nim}$ mutants to these compounds, although reduced, was not blocked completely, despite evidence suggesting that these compounds are capable of being transported by the choline permease (Robson et al., 1992).

We have shown previously that $\left[{ }^{3} \mathrm{H}\right] \mathrm{GlcNAc}$ is incorporated primarily into the chitin fraction of the cell wall when added to growing cultures of $F$. graminearum (Binks et al., 1990). Thus, incorporation of $\left[{ }^{3} \mathrm{H}\right] \mathrm{GlcNAc}$ into the mycelium of $F$. graminearum is a measure of the in vivo 
chitin synthase activity. Incorporation of the label was found to be approximately logarithmically linear. Addition of choline caused a $75 \%$ increase in the incorporation of radiolabel within $30 \mathrm{~s}$, after which the rate of incorporation continued at the same rate as that prior to choline addition. This rapid increase in in vivo chitin synthase activity may reflect the morphological transition induced by choline. Choline causes an increase in the hyphal extension rate of the mycelium and a reduction in branching frequency without affecting overall growth rate (Wiebe et al., 1989). Consequently, as choline does not affect overall growth but only the spatial distribution of the biomass, in vivo chitin synthase activity should not be affected by choline. Indeed, we found that the rate of in vivo chitin synthase activity was not significantly different after the addition of choline (Fig. 4). The transient increase in the in vivo chitin synthase activity immediately following the addition of choline may result from an initial increase in the extension rate of the hyphae induced by choline before the inhibition of branch frequency. The subsequent inhibition in branch frequency would then establish the rate of in vivo chitin synthase to that exhibited prior to choline addition.

The results presented in this study support the hypothesis that choline and related analogues are transported by a number of permeases and act within the hypha, probably via a common active component which then mediates the morphological response. The morphological effect of acetylcholine appears to involve an acetylcholinesterase, presumably acting at the cell surface. The choline produced would then be transported via the choline permease. The choline-induced alteration in mycelial morphology is elicited very rapidly (within $30 \mathrm{~s}$ ) and may involve an increase in hyphal extension rate before inhibition of hyphal branching. The mechanisms involved in regulating hyphal branching and hyphal extension by choline are unknown, although we have previously shown that cGMP elicits a similar morphological response in $F$. graminearum and acts synergistically with choline (Robson et al., 1991). In addition, we have shown that the inhibition of branch frequency by choline occurs independently of the stimulation of hyphal extension rate (Wiebe et al., 1992). In Neurospora crassa, mutants blocked in choline synthesis require exogenous choline for growth. Growth can also be supported by the addition of betaine aldehyde but not betaine. As betaine aldehyde is an intermediate between choline and betaine, it appears that the oxidation of betaine aldehyde to betaine is irreversible (Richardson \& Speed, 1969). If this is also true in F. graminearum, then betaine may act as a common intermediate in eliciting the morphological effects of choline and related analogues.

\section{REFERENCES}

Binks, P. R., Robson, G. D., Goosey, M. W., Humphreys, A. \& Trinci, A. P. J. (1990). Chitin synthesis in Fusarium graminearum and its inhibition by edifenphos. J Gen Microbiol 137, 615-620.

Kinney, A. J. \& Moore, T. S. (1988). Phosphatidylcholine synthesis in castor bean endosperm. I. Metabolism of L-serine. Plant Physiol 84, 78-81.

Li, Z., Haase, E. \& Brendel, M. (1991). Hyper-resistance to nitrogen mustard in Saccharomyces cerevisiae is caused by defective choline transport. Curr Genet 19, 423-427.

Richardson, M. \& Speed, D. J. (1969). Utilisation of betaine aldehyde by choline $\left(\mathrm{chol}^{-}\right)$mutants of Neurospora crassa. Arch Mikrobiol 66, 199-202.

Robson, G. D., Wiebe, M. G. \& Trinci, A. P. J. (1991). Exogenous cAMP and cGMP modulate branching in Fusarium graminearum. $J$ Gen Microbiol 137, 963-969.

Robson, G. D., Best, L. C., Wiebe, M. G. \& Trinci, A. P. J. (1992). Choline transport in Fusarium graminearum A3/5. FEMS Microbiol Lett 92, 247-252.

Robson, G. D., Wiebe, M. G. \& Trinci, A. P. J. (1994). Betaine transport in Fusarium graminearum. Mycol Res 98, 176-178.

Strange, R. S. \& Smith, H. (1971). A fungal growth stimulant in anthers which predisposes wheat to attack by Fusarium graminearum in vitro. Physiol Plant Patbol 1, 141-150.

Strange, R. S. \& Smith, H. (1978). Effect of choline, betaine and wheat-germ extract on growth of cereal pathogens. Trans Br Mycol Soc 70, 193-199.

Strange, R. S., Majer, J. R. \& Smith, H. (1974). The isolation and identification of choline and betaine as the two major components in anthers and wheat germ that stimulate Fusarium graminearum in vitro. Physiol Plant Pathol 4, 277-290.

Trinci, A. P. J. (1971). Influence of the peripheral growth zone on the radial growth rate of fungal colonies. J Gen Microbiol 67, 325-344.

Vogel, H. J. (1956). A convenient growth medium for Neurospora (medium N). Microb Genet Bull 13, 42-44.

Wiebe, M. G., Robson, G. D. \& Trinci, A. P. J. (1989). Effect of choline on the morphology, growth and phospholipid composition of Fusarium graminearum. J Gen Microbiol 135, 2155-2162.

Wiebe, M. G., Robson, G. D. \& Trinci, A. P. J. (1992). Evidence for the independent regulation of hyphal extension and branch initiation in Fusarium graminearum A3/5. FEMS Microbiol Lett 90, 179-184.

Received 22 November 1994; revised 21 February 1995; accepted 2 March 1995. 